\title{
\&YHE RECOGNITION OF METALLIC GOLD IN TISSUE SECTIONS
}

\author{
BY \\ H. E. HARDING \\ From the Department of Pathology, Sheffield University
}

(RECEIVED FOR PUBLICATION OCTOBER 30, 1952)

The use of radioactive gold $\left(\mathrm{Au}^{198}\right)$ for symptomatic treatment of some cases of advanced malignant disease is increasing (e.g., King, Spicer, Dowda, Bender, and Noel, 1952). The isotope, in a carrier of colloidal gold, when injected into the peritoneal or pleural cavity of ten diminishes the production of a malignant ascites or other effusion for several weeks or months. The gold is precipitated in fine granular aggregates that under the microscope resemble haemosiderin, being relatively insoluble, opaque to transmitted light, and persisting after incineration of the section. It does not give a prussian blue or other similar reaction. Colour reactions with organic "spot reagents" are only theoretically obtainable, since they depend on previous conversion of the gold to a soluble form, and it is obviously impracticable to treat sections with aqua regia. If recently injected the active gold can be recognized by autoradiography, but this method soon becomes useless, since the half-life of the isotope is about three days.

The nature of the granules can, however, be demonstrated in a negative way. If, immediately before staining, sections are treated with a solution of potassium cyanide the gold dissolves and

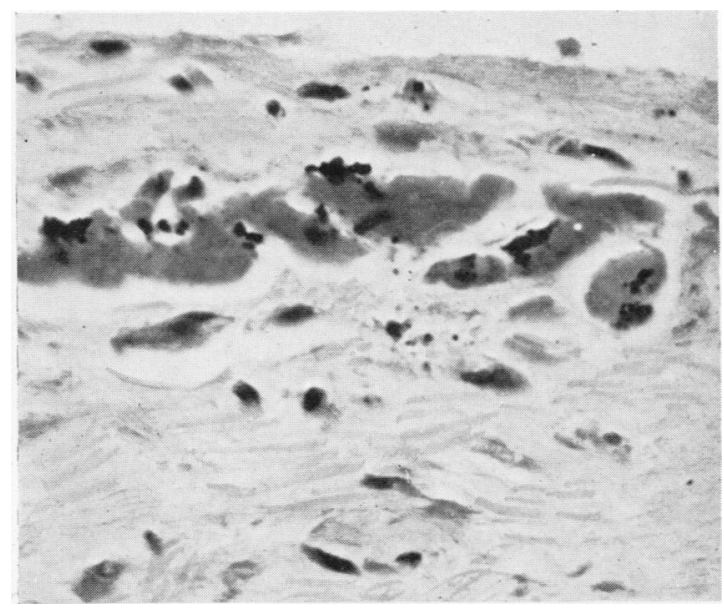

Fig. 1.-Edge of thickened peritoneum over caecum. Granules of gold lying close to deposits of fibrin. Haematoxylin and eosin the granules are no longer visible (Figs. 1 and 2). Almost all other opaque granules are unaffected by such treatment. A similar result is obtained with silver (McLaughlin, Barrie, Grout, and Harding, 1945), but the likelihood of confusion is very small, since it should be known whether silver was likely to be present (e.g., in a silver-polisher), and this latter metal tends to have a different distribution in the body and to be present in much finer granules. I have used $5 \% \mathrm{KCN}$ for 10 to 15 minutes, but much more dilute solutions, e.g., $0.3 \%$, are effective. The stronger solutions show some tendency to diminish the intensity of staining of the tissues with haematoxylin, but this is not a serious'matter. When granules of gold are present in reticulo-endothelial cells it is possible to remove this metal by cyanide and then demonstrate any particles of haemosiderin by the usual methods.

The photomicrographs were prepared by Mr. L. Reeves, F.I.M.L.T.

\section{REFERENCES}

King, E. R., Spicer, D. W., Dowda, F. W., Bender, M. A., and Noel, W. E. (1952). Amer. J. Roentgenol., 68, 413.

McLaughlin, A. I. G., Barrie, H. J., Grout. J. L. A., and Harding, H. E. (1945). Lancet, 1, 337.

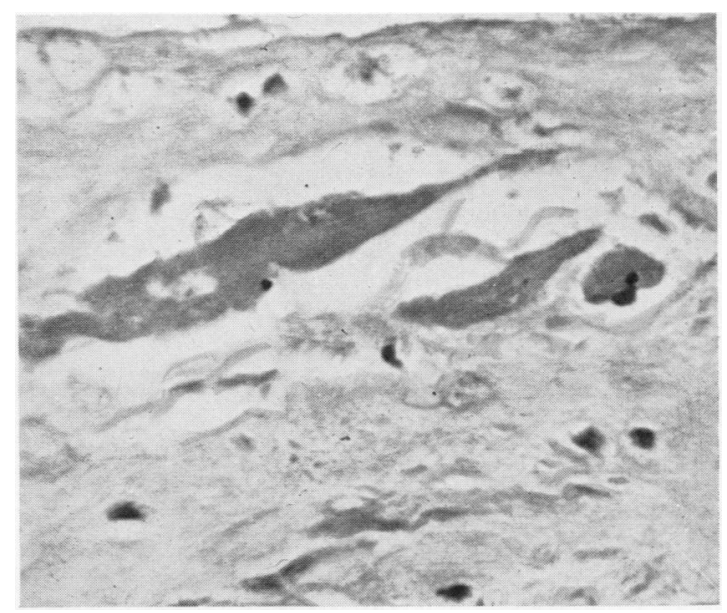

FIG. 2.-Duplicate section treated with $\mathrm{KCN}$ solution before staining. Note disappearance of granules. 